\title{
Low dose dinaciclib enhances doxorubicin-induced senescence in myeloma RPMI8226 cells by transformation of the p21 and p16 pathways
}

\author{
HAILONG TANG $^{1 *}$, LI XU $^{1 *}$, XUE LIANG $^{2}$ and GUANGXUN GAO ${ }^{1}$ \\ ${ }^{1}$ Department of Hematology, Xijing Hospital, Air Force Military Medical University; \\ ${ }^{2}$ School of Basic Medicine, Air Force Military Medical University, Xi'an, Shaanxi 710032, P.R. China
}

Received January 3, 2018; Accepted August 31, 2018

DOI: $10.3892 / \mathrm{ol} .2018 .9474$

\begin{abstract}
Multiple myeloma (MM) is a hematological malignancy that lacks a cure. However, novel combination therapy is a current anti-MM strategy. Doxorubicin (DOX) is a type of anthracycline which is a first-line chemotherapeutic for treating $\mathrm{MM}$ and induces senescence in many types of cancer. Dinaciclib is a potent, small molecule CDK inhibitor with promise for treating several types of cancer in I/II phase clinical trials. In the present study the anticancer effects and underlying mechanisms of dinaciclib combined with DOX in MM RPMI-8226 cells were investigated. Results indicated that DOX induced cell viability inhibition, cell cycle arrest and senescence. Furthermore, DOX resulted in increased alterations in DNA damage-related proteins such as p-ATM, p-Chk2, p-p53, p21 and $\gamma \mathrm{H} 2 \mathrm{AX}$, but not p16. Notably, the combination of dinaciclib and DOX inhibited cell growth and promoted senescence by transforming the suppressive effects of the ATM/Chk2/p53/p21 signaling pathway and enhancing the p16 signaling pathway. Thus, low-dose dinaciclib enhanced anti-MM effects mediated by DOX via transformation of p21-p16 signaling pathways, leading to accelerated senescence, but not apoptosis. The present findings suggest this approach may be a promising therapeutic strategy for the treatment of MM.
\end{abstract}

\section{Introduction}

Cellular senescence is a persistent cell-cycle arrest of actively proliferating cells that contributes to physiological and pathological processes including tumor suppression,

Correspondence to: Dr Guangxun Gao, Department of Hematology, Xijing Hospital, Air Force Military Medical University, 127 Changle Road, Xincheng, Xi'an, Shaanxi 710032, P.R. China E-mail: gaoguangxun@fmmu.edu.cn

\section{${ }^{*}$ Contributed equally}

Key words: dinaciclib, doxorubicin, DNA damage, senescence, multiple myeloma inflammatory response and tissue repair $(1,2)$. Senescence is not a chief strategy of cancer therapy but it may be a key antitumor response to counteract carcinogenic stress. Cellular senescence is a primary response against tumorigenesis, and senescent cells can be eliminated by the innate immune system (3). Chemotherapy-induced sublethal stress can accelerate a specific form of senescent phenotype in cells which is termed as stress-induced premature senescence (SIPS) (4), so we focused primarily on this approach.

Growth arrest of proliferating cells is often triggered by a persistent DNA damage response (DDR) and is classically executed by activation of the p16-Rb (retinoblastoma) and/or p53-p21 pathways (5-7). The ataxia telangiectasia mutated (ATM) protein acting as a damage sensor is recruited to damaged foci, stabilizing p53 and upregulating p53 transcriptional target p21 which inhibits cyclin-dependent kinase 2 (CDK2) and activates $\mathrm{Rb}$ to prevent entry into cell cycle (8-10). On the other hand, p16 inhibits CDK4 and CDK6, leading to inactivation of Rb to block cell cycle progression (6).

CDKs are a family of serine/threonine kinases that mainly play an important role in regulating cell cycle progression (11). The ultimate DDR targets are CDKs, key regulators of cell cycle progression (12). CDKs phosphorylate Rb tumor suppressor and the related 'pocket-proteins' p107 and p130 to maintain them in an inactive state. Phosphorylated (inactive) $\mathrm{pRb}$ and pocket-proteins facilitate E2F family transcription factors and promote genes expression related to DNA replication and cell division, eventually dysregulating cell cycle progression (13). In response of DNA replication stress, active p53 accelerates expression of the CDK inhibitor p21 which is an essential mediator of senescence (12). Except regulating cell cycle progression, the CDK family also has other physiological functions. For example, CDK5, known as a key regulator in neuronal cells, has also been found to participate in several physiological and pathological processes of extra neuronal activities, such as gene expression, cell migration, apoptosis, and senescence $(14,15)$. Disorder of cell cycle regulation and aberrant activation of CDKs occur in almost all human cancers $(16,17)$ and for the important role of CDKs in many biological processes commonly abnormally regulated in cancer cells, targeting CDKs chemically is considered to be a potential therapy for cancer treatment (18). 
Dinaciclib is a potent and specific inhibitor of CDK that interacts with the acetyl-lysine recognition site of bromodomains (19). Dinaciclib specifically targets CDK1/2/5/9 (20). Preclinical studies have shown it to be a potent growth inhibitor in a murine xenograft model of human cancers $(21,22)$ and a promoter of apoptosis in most cancer cells via suppression of $\mathrm{Rb}$ phosphorylation $(22,23)$. Here we investigated antineoplastic effects and mechanisms underlying dinaciclib plus DOX in preclinical models of multiple myeloma (MM).

\section{Materials and methods}

Cell culture and reagents. Human MM RPMI-8226 cell lines were generously gift from Dr. Xinliang Mao (The Cyrus Tang Hematology Center of Soochow University, Suzhou, China) and maintained in RPMI-1640 medium containing 10\% fetal calf serum (FBS), $100 \mathrm{U} / \mathrm{ml}$ penicillin and $100 \mathrm{ng} / \mathrm{ml}$ streptomycin. Cells were incubated at $37^{\circ} \mathrm{C}$ with $5 \% \mathrm{CO}_{2}$. Doxorubicin (DOX) was purchased from Sigma-Aldrich; Thermo Fisher Scientific, Inc. (Waltham, MA, USA; D1515). CDK inhibitor dinaciclib and ATM kinase inhibitor KU-55933 was purchased from Selleckchem (Houston, TX, USA; S2768 and S1092). Anti-ATM (ab32420), anti-p-ATM (S1981, ab81292), anti-Chk2 (ab109413), anti-p-Chk2 (T68, ab32148), anti-p53 (ab32389), anti- $\gamma$-H2AX (S139, ab2893), anti-p16 (ab08349), anti-p21 (ab109199) anti-CDK1 (ab133327), anti-CDK2 (ab32147), anti-CDK9 (ab76320) and anti-CDK5 (ab40773) were from Abcam (Cambridge, UK). Anti-p-p53 (S15) was from Immunoway. Anti-GAPDH (TA505454) was obtained from ZSBG-BIO (Beijing, China).

Cell viability assay. Cells were seeded in a 96-well plate (10,000 cells/well), and incubated with different concentrations of DOX in three wells for $24 \mathrm{~h}$. Cell viability was measured using an MTT kit (Sigma-Aldrich; Thermo Fisher Scientific, Inc.). Plates were detected at $570 \mathrm{~nm}$ on a microplate reader (DNM-9602; PERLONG, Beijing, China).

Cell cycle analysis. Cells were seeded in a 6-well plate (10x $10^{4}$ cells/well) and incubated with/without $100 \mathrm{nM}$ DOX for $24 \mathrm{~h}$. Cells were harvested and washed twice with cold PBS, then fixed with cold $70 \%$ ethanol for $1 \mathrm{~h}$ at $4^{\circ} \mathrm{C}$. Fixed cells were washed twice with $\mathrm{PBS}$ and resuspended with $0.4 \mathrm{ml}$ PBS containing PI (20 $\mu \mathrm{g} / \mathrm{ml}$; Sigma-Aldrich; Thermo Fisher Scientific, Inc.) and DNase-free RNaseA $(50 \mu \mathrm{g} / \mathrm{ml}$; Amresco LLC, Solon, OH, USA), and measured using flow cytometry after $30 \mathrm{~min}$ incubation at room temperature in the dark. Data were analyzed using Flowjo7.6 software (FlowJo LLC, Ashland, OR, USA).

Senescence associated $\beta$-galactosidase (SA- $\beta$-Gal) staining. Cells were treated as described and incubated on poly-L-lysine coated slides (P9155; Sigma-Aldrich; Thermo Fisher Scientific, Inc.) for $1 \mathrm{~h}$ at $37^{\circ} \mathrm{C}$. SA- $\beta$-Gal staining was conducted according to the manufacturers' instructions (cat. no. 9860) from Cell Signaling Technology, Inc. (Danvers, MA, USA). Cells positive for SA- $\beta$-Gal staining were quantified in 100 individual cells for each sample and analyzed $(n=3)$.
Immunofluorescence staining. Cells were treated as described and incubated on poly-L-lysine coated slides (P9155; Sigma-Aldrich; Thermo Fisher Scientific, Inc.) for $1 \mathrm{~h}$ at $37^{\circ} \mathrm{C}$. Then, cells were fixed in $4 \%$ paraformaldehyde for $20 \mathrm{~min}$ at room temperature, permeabilized with $0.5 \%$ Triton $\mathrm{X}-100$ for 15 min and blocked with $1 \%$ BSA dissolved in PBS for $1 \mathrm{~h}$. Cells were then incubated with anti- $\gamma$-H2AX (phospho-S139) (1:250; S139; ab2893) overnight at $4^{\circ} \mathrm{C}$, and labeled with TRICT-conjugated goat anti-rabbit secondary antibodies for $1 \mathrm{~h}$ at RT in the dark. Nuclei were stained with $0.1 \mu \mathrm{g} / \mathrm{ml}$ DAPI for 5 min. Cells were viewed under an Olympus (BX51; Olympus Corp., Tokyo, Japan) fluorescent microscope. $\gamma$-H2AX foci in nuclei were counted in 50 individual cells for each sample.

Western blot analysis. Cells were lysed in RIPA buffer and lysates were sonicated and then boiled and centrifuged (447.2 x g). Proteins were resolved with 8-15\% SDS-PAGE and transferred to PVDF membranes. After blocking, membranes were incubated with the indicated primary antibodies and then horseradish peroxidase-conjugated secondary antibodies (1:4,000; ab136817; Abcam). Blots were visualized using ECL and SuperSignal reagent (WBKLS0500; EMD Millipore, Billerica, MA, USA). Protein quantification was conducted using image $\mathrm{J}$ software.

Apoptosis assay. Cells were washed and stained with Annexin V-FITC and PI according to instructions (BD Bioscience, Franklin Lakes, NJ, USA). At least 1,000 cells were counted to calculate percent apoptotic in each sample. Cells with Annexin V+/PI- and Annexin V+/PI+ were defined as apoptotic cells.

Statistical analyses. Data were analyzed using GraphPad Prism 5 (GraphPad Software, Inc., La Jolla, CA, USA). Differences between multiple groups was confirmed using one-way analysis of variance followed by Tukey's post hoc test. The significance of differences between two groups was determined using the Student's t-test. Data were obtained from at least three independent experiments and $\mathrm{P}<0.05$ was considered to indicate a statistically significantly differenence.

\section{Results}

DOX inhibited proliferation, induced cell cycle arrest, and senescence in MM RPMI-8226 cells. Data from an MTT assay showed that DOX inhibited RMPI8226 cell growth in a dose-dependent manner (Fig. 1A). Subsequent experiments were conducted with $100 \mathrm{nM}$ DOX as a representative of DNA damaging agents to study the effects on MM cells. Next, we studied the effects of DOX on the cell cycle in RPMI-8226 cells. DNA content analysis with flow cytometry after DOX treatment for $24 \mathrm{~h}$ showed that DOX induced marked accumulation of $\mathrm{G} 2 / \mathrm{M}$ DNA content $(21.4 \%$ vs. $80.8 \%$; $\mathrm{P}<0.05)$ in RPMI-8226 cells compared to DMSO-treated control cells (Fig. 1B). Increased nuclear and cell body size, and staining for $\beta$-Gal, a molecular marker of cell senescence, revealed that DOX induces more senescence in RPMI-8226 cells at $24 \mathrm{~h}$ compared with DMSO (Fig. 1C). 
A

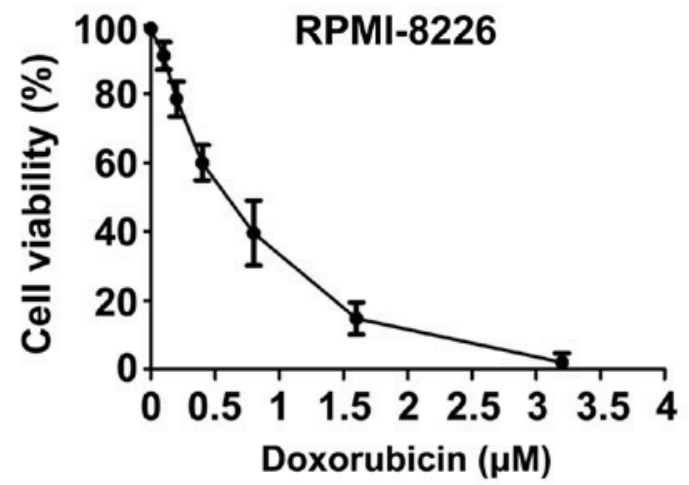

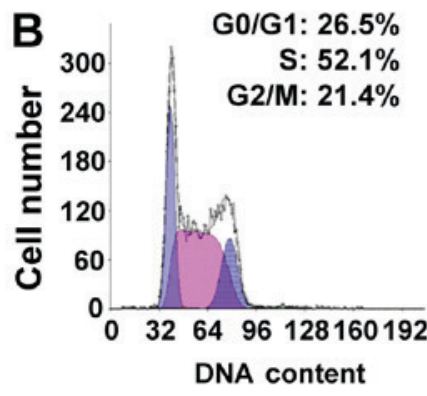

C

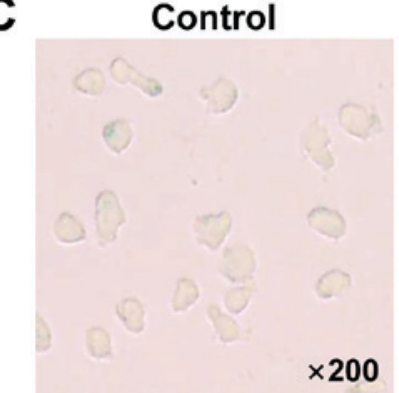

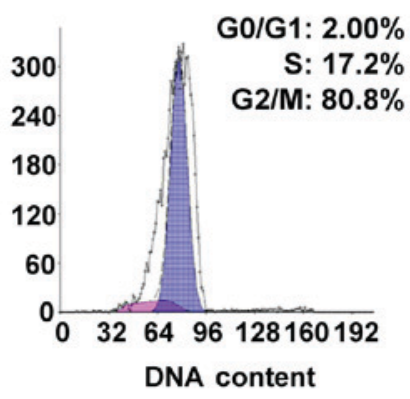

DOX

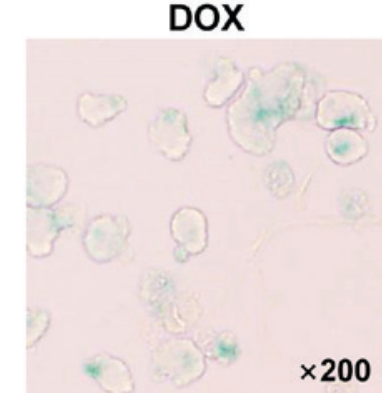

G0/G1

$\square \mathrm{S}$
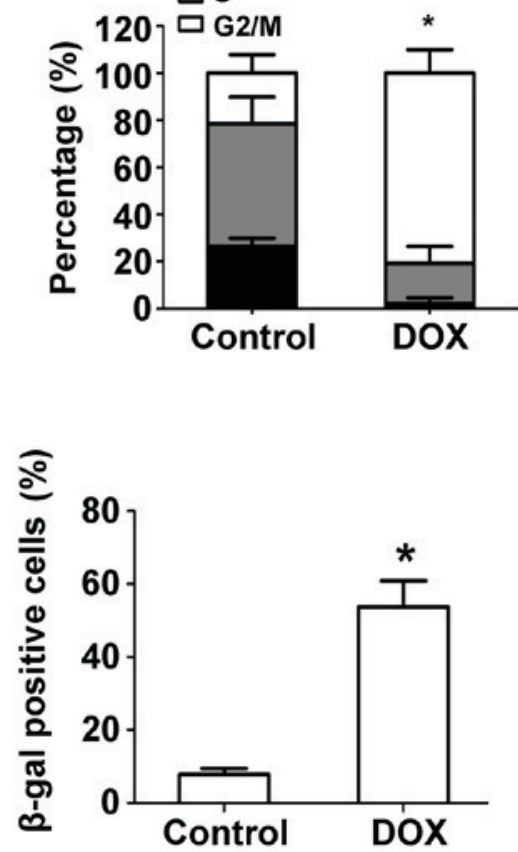

Figure 1. Effects of DOX on cell proliferation, cell cycle distribution and senescence in RPMI-8226 cells. (A) RPMI-8226 cells were incubated with various concentrations of DOX for $24 \mathrm{~h}$, cell proliferation was detected by MTT assay. RPMI-8226 cells were incubated with/without $100 \mathrm{nM}$ DOX for $24 \mathrm{~h}$. (B) Cell cycle distribution was measured using flow cytometry. Differences of percentages of cell cycle phase was confirmed using one-way analysis of variance followed by Tukey's post hoc test. "P $<0.05$ vs. control. (C) Senescence-associated $\beta$-gal was measured using X-Gal staining, and dark blue perinuclear staining marks senescent cells. Data were obtained from at least three independent experiments. The significance of differences between two groups was determined by Student's t-test. "P<0.05 vs. control. DOX, doxorubicin; MTT, multiple myeloma.

Senescence of RPMI-8226 cells caused by DOX accompanied with DSBs and activation of ATM/Chk2/p53/p21 signaling. To investigate whether DOX-induced senescence of MM cells and DNA damage as well as the pathway activated, immunofluorescent staining of $\gamma-\mathrm{H} 2 \mathrm{AX}$ and Western blot was used. Data show that DOX induced $\gamma-\mathrm{H} 2 \mathrm{AX}$ foci formation in the nucleus in RPMI-8226 cells (Fig. 2A). After treatment with DOX, an intense ATM phosphorylation response was observed and phosphorylation level of p53 and Chk2 was also elevated, resulting in stabilization and accumulation of p53 (24). Expression of the CDK inhibitor p21 was also stimulated by DOX induced oncogenic stress but not p16 (Fig. 2B and C). Thus, cell cycle exit and senescence after DOX exposure in RPMI-8226 cells was accompanied by DNA damage and activation of the ATM/Chk2/p53/p21 pathway, but not the p16 pathway.

Low-dose dinaciclib increased DOX-induced senescence of MM RPMI-8226 cells but not apoptosis. Dinaciclib has shown promise for treating non-small cell lung cancer, breast cancer and MM in some I/II phase clinical trials (18) but combination therapy deserves attention as a novel cancer treatment strategy. To evaluate anti-MM effects of dinaciclib plus DOX, we incubated RPMI-8226 cells with various concentrations of dinaciclib for $24 \mathrm{~h}$ and expression of CDK1/2/5/9 was measured. The minimum dose $(5 \mathrm{nM})$ of dinaciclib that decreased CDK5 was chosen for subsequent experiments to regulate expression of related proteins regardless of other involved CDKs as well as reduce toxicity clinically (Fig. 3A). Cell viability, apoptosis, and senescence were measured in RPMI-8226 cells and data showed that dinaciclib enhanced DOX-induced inhibition of cell viability (Fig. 3B), whereas apoptosis did not change (Fig. 3C). Co-treatment induced more senescent phenotype (Fig. 3D). We speculated that low-dose dinaciclib elevated anti-MM activity with DOX by promoting senescence but not apoptosis.

Dinaciclib transforms cells from the p21 to the p16 pathway in the senescence model induced by DOX in myeloma RPMI-8226 cells. Now that dinaciclib enhanced DOX-induced 
A D-H2AX
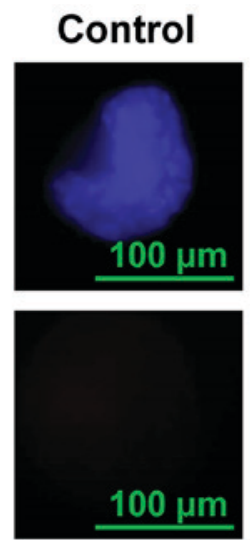

Merge

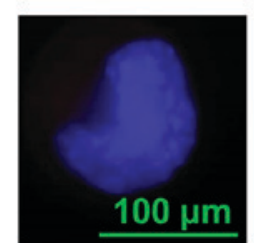

DOX
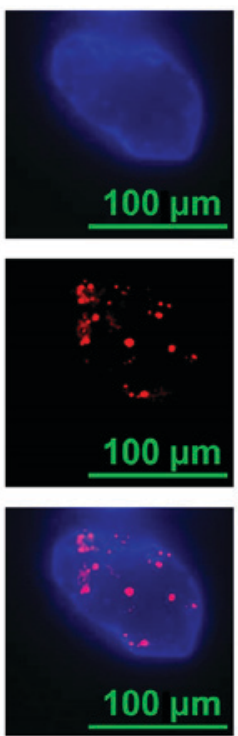

B
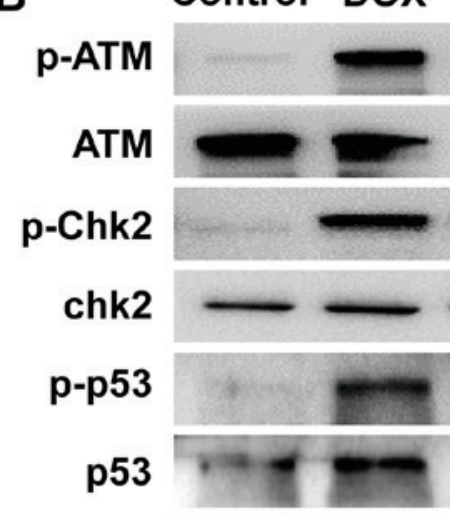

p21

p16

GAPDH
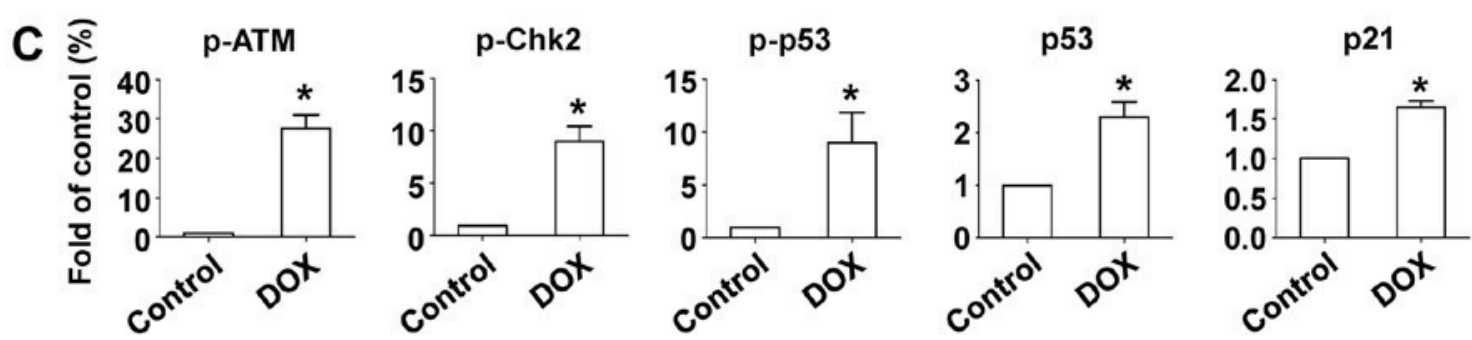

Figure 2. Effects of DOX on $\gamma$-H2AX foci formation and expression of DNA damage related proteins in RPMI-8226 cells. RPMI-8226 cells were incubated with/without $100 \mathrm{nM}$ DOX for $24 \mathrm{~h}$. (A) Cells were fixed and stained with $\gamma$-H2AX (red) and DAPI (blue) at the indicated time points. (B) RPMI-8226 cells treated with/without $100 \mathrm{nM}$ DOX for $24 \mathrm{~h}$ were extracted and proteins were incubated with primary antibodies against p-ATM, ATM, p-Chk2, Chk2, p-p53, p53, p21 and p16. GAPDH was used as a loading control. (C) Protein quantification was conducted and data were shown with fold of control. Data were obtained from at least three independent experiments. The significance of differences between two groups was determined by Student's t-test. ${ }^{*} \mathrm{P}<0.05$ vs. control. DOX, doxorubicin; MTT, multiple myeloma; ATM, ataxia telangiectasia mutated.

A

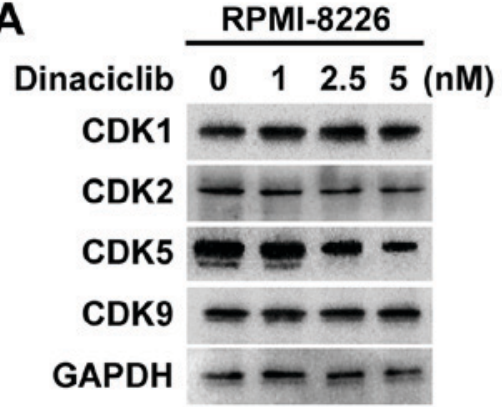

D Control

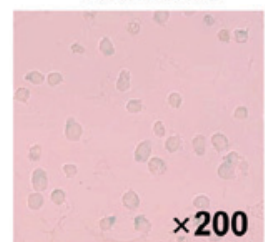

B

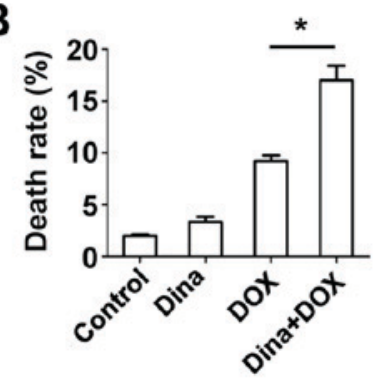

DOX
C

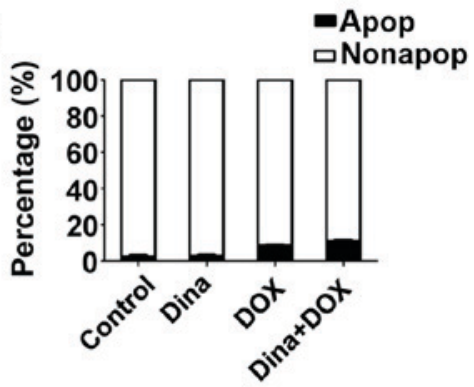

Figure 3. Dinaciclib additively enhanced anti-MM effect of DOX by augmenting DOX-triggered senescence rather than apoptosis. (A) RPMI-8226 cells treated with dinaciclib for $24 \mathrm{~h}$ were extracted and proteins were incubated with primary antibodies against CDK1/2/5/9. GAPDH was used as a loading control. RPMI-8226 cells were treated with DMSO, $100 \mathrm{nM}$ DOX, $5 \mathrm{nM}$ dinaciclib, or both for 24 h. Cell viability (B), apoptotic cells (C) and senescence-associated $\beta$-gal (D) was measured. Data were obtained from at least three independent experiments. Differences between multiple groups was confirmed using one-way analysis of variance followed by Tukey's post hoc test. " $\mathrm{P}<0.05$ as indicated. DOX, doxorubicin; CDK, cyclin-dependent kinase. 
A

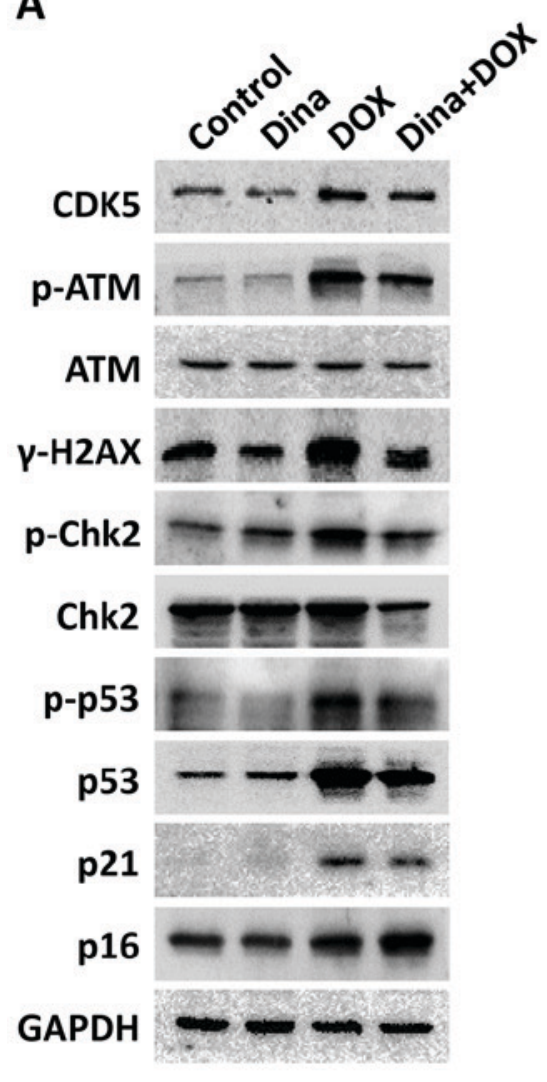

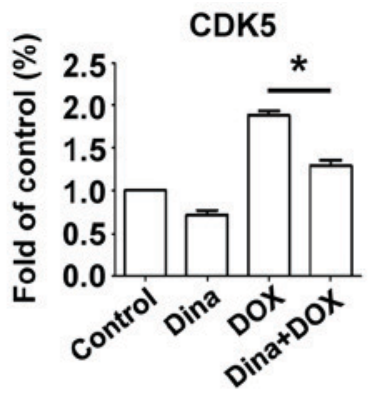

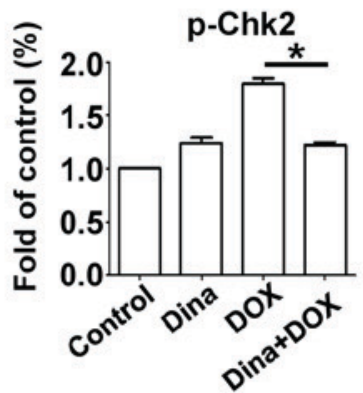

p21

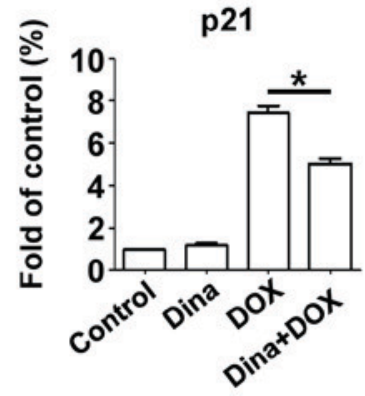

p-ATM

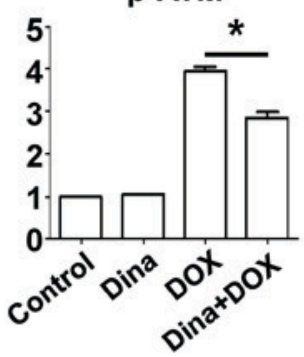

p-p53

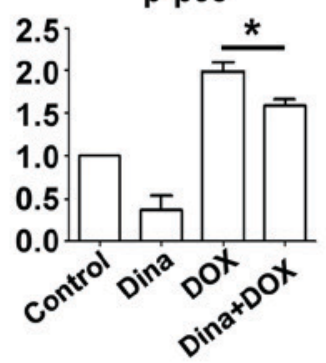

p16

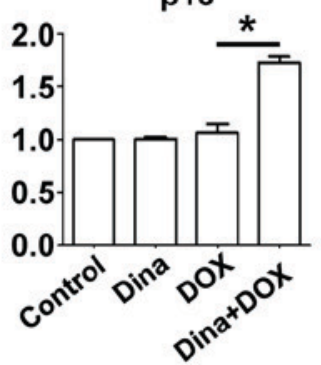

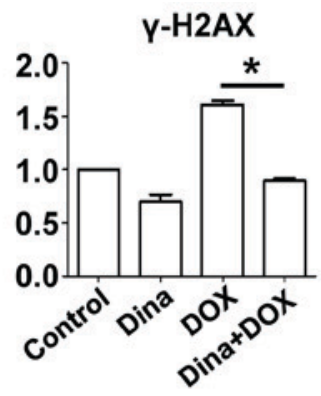

p53

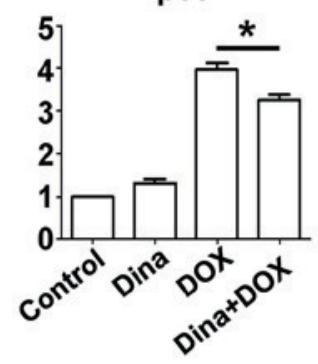

C
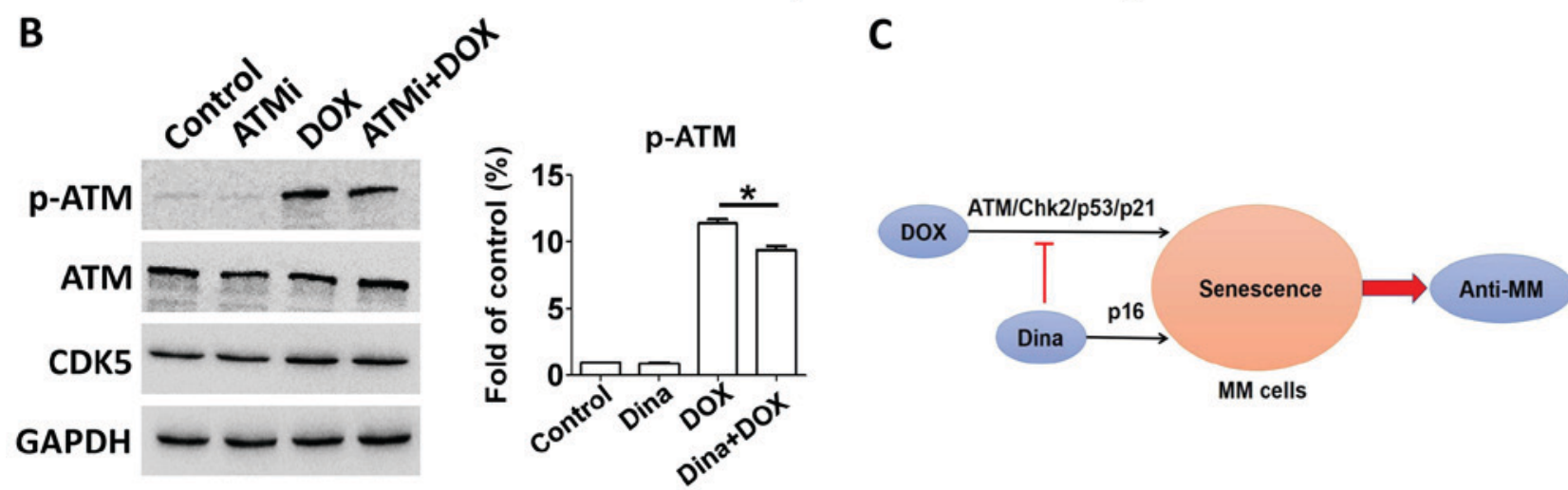

Figure 4. Dinaciclib transforms the p21 to the p16 pathway in a senescence model induced by DOX in MM RPMI-8226 cells. (A) RPMI- 8226 cells were treated with DMSO, $100 \mathrm{nM}$ DOX, $5 \mathrm{nM}$ dinaciclib, or DOX and dinaciclib for $24 \mathrm{~h}$. Cells were extracted and proteins were incubated with primary antibodies against p-ATM, ATM, $\gamma$-H2AX, p-Chk2, Chk2, p-p53, p53, p21 and p16. GAPDH was used as a loading control. Protein quantification was conducted. (B) RPMI-8226 cells were treated with DMSO, $10 \mu \mathrm{M}$ KU-55933, $100 \mathrm{nM}$ DOX, or DOX and KU-55933 for $24 \mathrm{~h}$. Cells were extracted and proteins were incubated with primary antibodies against p-ATM, ATM, CDK5. GAPDH was used as a loading control. Protein quantification was conducted. (C) Low-dose of dinaciclib enhanced anti-MM effects mediated by DOX via transformation of the p21-p16 pathways, accelerating senescence rather than apoptosis induced by DOX. Data were obtained from at least three independent experiments. Differences between multiple groups was confirmed using one-way analysis of variance followed by Tukey's post hoc test. "P $<0.05$ as indicated. MTT, multiple myeloma; DOX, doxorubicin; ATM, ataxia telangiectasia mutated; DOX, doxorubicin; CDK, cyclin-dependent kinase.

senescence in RPMI-8226 cells, it was easy to assume that whether this phenomenon was accompanied by elevated p21 expression. Interestingly western blot for expression of ATM-related proteins showed that dinaciclib partly reduced phosphorylated ATM and phosphorylation of ATM downstream Chk2/p53 proteins and level of p21 (Fig. 4A). We then measured activation of alternative executor p16 and its expression of combination was greater compared with DOX alone in RPMI-8226 cells (Fig. 4A), suggesting that dinaciclib enhanced DOX triggered senescence of RPMI- 8226 cells by switching from $\mathrm{p} 21$ to p16 pathways.

Next we explored the preliminary mechanism of this transformation. Our data showed that dinaciclib inhibited expression of CDK5 and phosphorylation of ATM. After incubating RPMI-8226 cells with $10 \mu \mathrm{M}$ ATM inhibitor KU-55933 and DOX for $24 \mathrm{~h}$, we measured expressions of CDK5 and p-ATM 
and found inhibition of ATM kinase did not lead to change of CDK5 expression (Fig. 4B). Hence targeting CDK5 restrained DOX-triggered upregulation of p21 protein by orchestrating ATM phosphorylation, whereas meanwhile p16 expression was elevated. Given that inhibiting CDK5 or upregulating p16 brought about decreased phosphorylation of $\mathrm{Rb}$ protein $(6,13)$. We speculated that inhibition of CDK5 might lead to an increased expression of p16 protein due to the negative feedback regulation.

\section{Discussion}

MM is neoplastic and characterized by clinical symptoms, which means its pathogenesis is not clear and a cure does not exist. At this time, MM treatment chiefly consists of proteasome inhibitors, immunomodulatory drugs (IMiDs), epigenetic drugs, monoclonal antibody and immunotherapy. The emergence of so many new drugs on the one hand brings gospels to more and more MM patients; on the other hand, it also indicates that $\mathrm{MM}$ is still an incurable disease and the development of new drug targets is always in progress. Better MM treatment may arise from combinations of existing chemotherapeutics. In this study we focused on a new CDK inhibitor dinaciclib and evaluated its effects of combination with DOX on MM.

DNA damaging agents combined with proteasome inhibitor (such as bortezomib) are highly effective in the treatment of newly diagnosed or relapsed MM (25). Regardless, DNA damage agents are still important drugs in MM treatment, even if new classes of drugs are widely used. DNA damaging agents can trigger DDR in various types of cancer cells and activate multiple signaling pathways to inhibit cell growth, manifested as senescence, apoptosis or necrosis. While senescent cells are metabolic, antineoplastic roles of senescence in cancer treatment is to inhibit proliferation of cancer cells and activate the immune system to eliminate the senescent cells. We proved that low dose of DOX, a topoisomerase II inhibitor, generated DNA double strand breaks (DSBs) in MM RPMI-8226 cells, evidenced by the formation of $\gamma-\mathrm{H} 2 \mathrm{AX}$ foci in the nucleus. And DOX also enhanced phosphorylation level of ATM protein, mediated downstream phosphorylation cascade of ATM, including Chk2 and p53, leading to an increasing expression of p21, an inhibitor of CDK. Low dose of DOX lead to cell cycle arrest and senescent phenotypes in RPMI-8226 cells. However, the p16-pRb pathway was not activated.

Most tumors are highly proliferative due to abnormal expression of CDKs and cell cycle check point dysregulation. So, targeting CDKs may be an antitumor strategy. Dinaciclib, a specific inhibitor of CDK1/2/5/9, has been proven effective in an early clinical trial as monotherapy for treating refractory and relapsed MM (20). Thus, we focused on the efficacy of DOX and dinaciclib. To evaluate the effect of dinaciclib on its target molecules, we incubated RPMI-8226 cells with different concentrations of dinaciclib for $24 \mathrm{~h}$ and detected expression of CDK1/2/5/9. We found that low dose $(5 \mathrm{nM})$ of dinaciclib could only influence activity of CDK5 but not the others. So subsequent experiments about the effect of pharmacological inhibition of CDK5 combined with DOX on RPMI-8226 cells were conducted.

Over the last decade Cdk5 has been shown to be essential for neuronal functions (26), but it may have roles outside the nervous system. Cdk5 is generally dysregulated in various types of cancer (15), and targeting it may become a novel and promising therapeutic strategy for cancer treatment. CDK5 participates in DDR by phosphorylating ATM and downstream proteins (27-31), so we treated RPMI-8226 cells with the lowest concentration of dinaciclib that caused a decline in CDK 5 and DOX for $24 \mathrm{~h}$, assuming dinaciclib could influence DDR and senescence induced by DOX in MM cells. Results showed that compared to DOX alone, the combination brought about decreased cell viability and increased senescence in RPMI-8226 cells, but apoptosis was unchanged. Thus, dinaciclib at least partly strengthened the effect with DOX against MM by enhancing DOX-triggered senescence rather than apoptosis. Given that DOX-induced senescence of RPMI-8226 cells was accompanied by activation of the p21 pathway, we speculated whether dinaciclib accelerated DOX-induced senescence of RPMI-8226 cells by enhancing expression of p21 protein. So we measured expression of related proteins in ATM/Chk2/p53/p21 pathway after combination treatment in RPMI-8226 cells and unexpectedly we noted that p21 protein slightly decreased. It was speculated that there could be alternative pathway involved in this senescent model. And elevated p16 expression was confirmed in combination dinaciclib with DOX compared to DOX alone in RPMI-8226 cells. Thus, dinaciclib partly enhanced senescence induced by DOX in RPMI-8226 cells via a switch from the p21 to the p16 pathway.

In our study, phosphorylated ATM declined simultaneously with inhibition of expression of CDK5. According to other work, CDK 5 participates in DDR by phosphorylating ATM. To study this effect in MM cells, we incubated RPMI-8226 cells with KU-55933 to block ATM kinase, and DOX for $24 \mathrm{~h}$ simultaneously, andassayed the expression of CDK5. Data showed that the expression of phosphorylated ATM decreased and expression of CDK5 was unchanged after inhibiting ATM kinase activity. The result revealed that CDK5 participated in ATM-related DDR at least partly by regulating phosphorylated ATM. Targeting CDK5 restrained DOX-triggered upregulation of p21 protein by orchestrating ATM phosphorylation, whereas meanwhile p16 expression was elevated. But how does this transformation work? It had been proved that both CDK5 and p16 are upstream regulators of $\mathrm{Rb}$ protein. Inhibiting CDK 5 or upregulating p16 brought about decreased phosphorylation of $\mathrm{Rb}$ protein $(6,13)$. We speculated that after inhibition of CDK5 activity by dinaciclib, the phosphorylation level of $\mathrm{Rb}$ protein declined, leading to an increased expression of p16 protein due to the negative feedback regulation. And the deep mechanism is our subsequent research direction.

Besides both RPMI-8226 and H929 cell lines were tested in our study, but H929 cells failed to gain good results as well as RPMI-8226 cells (data not shown), so we speculate that the conclusions of this study may be associated with type of MM cell line. In future studies we will order other MM cell lines from ATCC, such as U226 or MM.1S to further verify our conclusion.

In conclusion, dinaciclib enhances anti-MM effects of DOX by transformation of the p21-p16 pathways, accelerating senescence rather than apoptosis induced by DOX in MM RPMI-8226 cells (Fig. 4C). These data may help to provide tailored treatment for MM patients. 


\section{Acknowledgements}

Not applicable.

\section{Funding}

The present study was supported by the Social Development Science and Technology Fund of Shaanxi Province (grant no. 2016SF-071) and the Natural Science Foundation of Shaanxi Province (grant no. 2017JM8025).

\section{Availability of data and materials}

All data generated or analyzed in this study are included in this manuscript.

\section{Authors' contributions}

HT and GG designed the study. HT, LX and XL conducted the experiments and performed the statistical analysis. HT and GG reviewed and provided final approval of the version to be published. All authors read and approved the manuscript.

\section{Ethics approval and consent to participate}

Not applicable.

\section{Patient consent for publication}

Not applicable.

\section{Competing interests}

The authors declare that they have no competing interests.

\section{References}

1. Rodier F and Campisi J: Four faces of cellular senescence. J Cell Biol 192: 547-556, 2011

2. He S and Sharpless NE: Senescence in health and disease. Cell 169: 1000-1011, 2017.

3. Xue W, Zender L, Miething C, Dickins RA, Hernando E, Krizhanovsky V, Cordon-Cardo C and Lowe SW: Senescence and tumour clearance is triggered by $\mathrm{p} 53$ restoration in murine liver carcinomas. Nature 445: 656-660, 2007.

4. Brack C, Lithgow G, Osiewacz $\mathrm{H}$ and Toussaint O: EMBO WORKSHOP REPORT: Molecular and cellular gerontology Serpiano. Switzerland, September 18-22, 1999. EMBO J 19: 1929-1934, 2000.

5. Campisi J: Aging, cellular senescence, and cancer. Annu Rev Physiol 75: 685-705, 2013.

6. Childs BG, Durik M, Baker DJ and van Deursen JM: Cellular senescence in aging and age-related disease: From mechanisms to therapy. Nat Med 21: 1424-1435, 2015.

7. Muñoz-Espín D and Serrano M: Cellular senescence: From physiology to pathology. Nat Rev Mol Cell Biol 15: 482-496, 2014.

8. Alcorta DA, Xiong Y, Phelps D, Hannon G, Beach D and Barrett JC: Involvement of the cyclin-dependent kinase inhibitor p16 (INK4a) in replicative senescence of normal human fibroblasts. Proc Natl Acad Sci USA 93: 13742-13747, 1996.

9. Takahashi A, Ohtani N, Yamakoshi K, Iida S, Tahara H, Nakayama K, Nakayama KI, Ide T, Saya H and Hara E: Mitogenic signalling and the p16INK4a-Rb pathway cooperate to enforce irreversible cellular senescence. Nat Cell Biol 8: 1291-1297, 2006.

10. Beauséjour CM, Krtolica A, Galimi F, Narita M, Lowe SW, Yaswen P and Campisi J: Reversal of human cellular senescence: Roles of the p53 and p16 pathways. EMBO J 22: 4212-4222, 2003.
11. Satyanarayana A and Kaldis P: Mammalian cell-cycle regulation: Several Cdks, numerous cyclins and diverse compensatory mechanisms. Oncogene 28: 2925-2939, 2009.

12. Gire V and Dulic V: Senescence from G2 arrest, revisited. Cell Cycle 14: 297-304, 2015.

13. Burkhart DL and Sage J: Cellular mechanisms of tumour suppression by the retinoblastoma gene. Nat Rev Cancer 8: 671-682, 2008.

14. Arif A: Extraneuronal activities and regulatory mechanisms of the atypical cyclin-dependent kinase Cdk5. Biochem Pharmacol 84: 985-993, 2012.

15. Contreras-Vallejos E, Utreras E and Gonzalez-Billault C: Going out of the brain: Non-nervous system physiological and pathological functions of Cdk5. Cell Signal 24: 44-52, 2012.

16. Konecny GE: Cyclin-dependent kinase pathways as targets for women's cancer treatment. Curr Opin Obstet Gynecol 28: 42-48, 2016.

17. Hall M and Peters G: Genetic alterations of cyclins, cyclin-dependent kinases, and Cdk inhibitors in human cancer. Adv Cancer Res 68: 67-108, 1996.

18. Chen XX, Xie FF, Zhu XJ, Lin F, Pan SS, Gong LH, Qiu JG, Zhang WJ, Jiang QW, Mei XL, et al: Cyclin-dependent kinase inhibitor dinaciclib potently synergizes with cisplatin in preclinical models of ovarian cancer. Oncotarget 6: 14926-14939, 2015.

19. Martin MP, Olesen SH, Georg GI and Schönbrunn E: Cyclin-dependent kinase inhibitor dinaciclib interacts with the acetyl-lysine recognition site of bromodomains. ACS Chem Biol 8: 2360-2365, 2013.

20. Kumar SK, LaPlant B, Chng WJ, Zonder J, Callander N, Fonseca R, Fruth B, Roy V, Erlichman C and Stewart AK; Mayo Phase 2 Consortium: Dinaciclib, a novel CDK inhibitor, demonstrates encouraging single-agent activity in patients with relapsed multiple myeloma. Blood 125: 443-448, 2015.

21. Feldmann G, Mishra A, Bisht S, Karikari C, Garrido-Laguna I, Rasheed Z, Ottenhof NA, Dadon T, Alvarez H, Fendrich V, et al: Cyclin-dependent kinase inhibitor Dinaciclib (SCH727965) inhibits pancreatic cancer growth and progression in murine xenograft models. Cancer Biol Ther 12: 598-609, 2011.

22. Parry D1, Guzi T, Shanahan F, Davis N, Prabhavalkar D, Wiswell D, Seghezzi W, Paruch K, Dwyer MP, Doll R, et al: Dinaciclib (SCH 727965), a novel and potent cyclin-dependent kinase inhibitor. Mol Cancer Ther 9: 2344-2353, 2010.

23. Fu W, Ma L, Chu B, Wang X, Bui MM, Gemmer J, Altiok S and Pledger WJ: The cyclin-dependent kinase inhibitor SCH 727965 (dinacliclib) induces the apoptosis of osteosarcoma cells. Mol Cancer Ther 10: 1018-1027, 2011.

24. Stracker TH and Petrini JH: The MRE11 complex: Starting from the ends. Nat Rev Mol Cell Biol 12: 90-103, 2011.

25. Kumar SK, Rajkumar V, Kyle RA, van Duin M, Sonneveld P, Mateos MV, Gay F and Anderson KC: Multiple myeloma. Nat Rev Dis Primers 3: 17046, 2017.

26. Dhavan R and Tsai LH: A decade of CDK5. Nat Rev Mol Cell Biol 2: 749-759, 2001.

27. Ajay AK, Upadhyay AK, Singh S, Vijayakumar MV, Kumari R, Pandey V, Boppana R and Bhat MK: Cdk5 phosphorylates non-genotoxically overexpressed p53 following inhibition of PP2A to induce cell cycle arrest/apoptosis and inhibits tumor progression. Mol Cancer 9: 204, 2010.

28. Ogara MF, Belluscio LM, de la Fuente V, Berardino BG, SonzogniSV,Byk L, Marazita Mand CánepaET: CDK5-mediated phosphorylation of p19INK4d avoids DNA damage-induced neurodegeneration in mouse hippocampus and prevents loss of cognitive functions. Biochim Biophys Acta 1843: 1309-1324, 2014.

29. Tian B, Yang Q and Mao Z: Phosphorylation of ATM by Cdk5 mediates DNA damage signalling and regulates neuronal death. Nat Cell Biol 11: 211-218, 2009.

30. Huang E, Qu D, Zhang Y, Venderova K, Haque ME, Rousseaux MW, Slack RS, Woulfe JM and Park DS: The role of Cdk5-mediated apurinic/apyrimidinic endonuclease 1 phosphorylation in neuronal death. Nat Cell Biol 12: 563-571, 2010.

31. Qu D, Rashidian J, Mount MP, Aleyasin H, Parsanejad M, Lira A, Haque E, Zhang Y, Callaghan S, Daigle M, et al: Role of Cdk5-mediated phosphorylation of Prx 2 in MPTP toxicity and Parkinson's disease. Neuron 55: 37-52, 2007. 